\title{
Numerical models to predict the creep behaviour of brickwork
}

\author{
A. Taliercio \\ Dept. of Structural Engineering, Politecnico di Milano, Milan, Italy
}

\begin{abstract}
The numerical modelling of the time evolution of stresses and strains in brickwork under sustained loads is dealt with, within the framework of linear viscoelasticity. Finite element analyses were carried out, using three different masonry models: a simplified 2D layered model, and two 3D models (one for header bond masonry; one for Flemish bond masonry). The creep behaviour of the component materials (bricks and mortar) was defined according to experimental data available in the literature. These results were best fitted by Prony series, and the obtained creep laws were employed to carry out FE analyses of the masonry walls with different textures. Owing to the different mechanical response of the components to sustained loads, the stress and strain distribution in the wall changes in time and differs from that at the beginning of the loading process. The different behaviour of the two considered brick patterns is pointed out. The possibility of applying the simplified layered model instead of refined 3D models to predict the time evolution of stresses and strains is discussed.
\end{abstract}

Keywords: masonry, creep, linear viscoelasticity, header bond, Flemish bond.

\section{Introduction}

The application of sustained loads on masonry structural elements induces creep phenomena, accompanied by a redistribution of stresses and strains. Under service loads, strains usually stabilize after a given time. On the contrary, damage effects (i.e. microcracks) induced by heavy loads can coalesce and grow, bringing the structural element to failure. In the former case, anyway, the longterm stress and strain state can differ, even considerably, from the short-term one. 
An overview of the mechanical (rheological) models currently employed to describe the creep behaviour of masonry and other quasi-brittle materials can be found in [1]. In the present work, only the response of brickwork under service loads will be investigated: accordingly, mortar joints and units will be assumed to have a linear viscoelastic behaviour.

The layout of this paper is as follows. First the mathematical modeling of the creep behaviour of the masonry constituents is dealt with, within the framework of linear viscoelasticity (Sec. 2.1). The selected model is calibrated using creep test data obtained by other authors on brick and mortar samples (Sec. 2.2). 3D finite element models of two walls characterized by different brick textures are developed and subjected to numerical creep tests; a simplified 2D layered model is also considered (Sec. 3.1). The time evolution of the wall displacements predicted by the different models is compared with the experimental one to validate their reliability (Sec. 3.2). The influence of the brick pattern on the time evolution of the stresses is analyzed in Sec. 4; comparisons with the predictions given by the simplified 2D model are also added. Finally, the main findings of the work are summarized in Sec. 5 .

\section{Mathematical modelling of experimental creep tests}

\subsection{Mathematical modelling}

Within the design stress range, the components of brick masonry (units and mortar) can be individually assumed to have a linear viscoelastic behaviour. Provided that loads are applied when the structural element is sufficiently hardened, the mechanical properties of the brickwork constituents are sensibly invariant in time, so that they can be assumed to be non-ageing materials.

For linear viscoelastic non-ageing materials subjected to any prescribed uniaxial stress history starting at time $t=0$, according to Boltzmann's superposition principle the strain at any time $t>0$ is given by (see e.g. [2])

$$
\varepsilon(t)=\int_{0}^{t} J(t-\tau) \dot{\sigma}(\tau) d \tau
$$

where $J$ is called creep compliance. Provided that time derivatives are intended in the distribution's sense, stress histories including jumps can also be taken into account in eq. (1).

Conversely, if a material element is subjected to a prescribed uniaxial strain history, the stress-strain law can be written as

$$
\sigma(t)=\int_{0}^{t} E(t-\tau) \dot{\varepsilon}(\tau) d \tau
$$

$E$ is called relaxation function, and is often denoted by $R$ in the literature, accordingly. The creep compliance is the 'inverse' of the relaxation function in the sense of Carson transforms [2]. 
Based on the so-called generalized Maxwell rheological model [3], consisting of linear springs and dashpots, the relaxation function can be expressed in the form

$$
E(t)=E_{\infty}+\sum_{i=1}^{n} E_{i} e^{-t / \tau_{i}}
$$

where $E_{\infty}$ is the equilibrium (or delayed) modulus, $E_{i}$ are called relaxation strengths and $\tau_{i}$ relaxation times. Note that the instantaneous modulus is $E_{0}=E_{\infty}$ $+\sum E_{i}$. The series expression in eq. (3) is usually referred to as a Prony (or Dirichlet) series.

For isotropic materials subjected to 3D strain histories, the stress-strain law can be obtained assuming that eq. (2) holds both for the hydrostatic stress $(p \boldsymbol{I})$ and the deviatoric stress $(\boldsymbol{S})$ :

$$
\boldsymbol{\sigma}(t) \equiv \boldsymbol{S}(t)+p(t) \boldsymbol{I}=\int_{0}^{t} 2 G(t-\tau) \dot{\boldsymbol{\eta}}(\tau) d \tau+\boldsymbol{I} \int_{0}^{t} K(t-\tau) \dot{e}(\tau) d \tau,
$$

where $e(t)$ (resp. $\eta(t))$ is the volumetric strain (resp. the deviatoric strain) at time $t, K$ (resp. $G$ ) is the bulk (resp. the shear) relaxation modulus, and $\boldsymbol{I}$ is the identity tensor. Both relaxation moduli are assumed to be of the form (3), namely

$$
K(t)=K_{\infty}+\sum_{i=1}^{n} K_{i} e^{-t / \tau_{i}^{K}}, G(t)=G_{\infty}+\sum_{i=1}^{n} G_{i} e^{-t / \tau_{i}^{G}},
$$

where $K_{\infty}$ (resp. $G_{\infty}$ ) is the long-term bulk (resp. shear) modulus. In general, the relaxation times $\tau_{i}^{K}$ and $\tau_{i}^{G}$ might differ from each other; for the sake of simplicity, $\tau_{i}^{K}=\tau_{i}^{G} \equiv \tau_{i}(i=1 \ldots n)$ will be assumed from here onwards.

Focusing on the material shear behaviour, the deviatoric stress at any time $t$ can be expressed as

$$
\boldsymbol{S}(t)=\int_{0}^{t} 2\left(G_{\infty}+\sum_{i=1}^{n} G_{i} e^{(\tau-t) / \tau_{i}}\right) \dot{\boldsymbol{\eta}}(\tau) d \tau=2 G_{0}\left(\boldsymbol{\eta}(t)-\sum_{i=1}^{n} g_{i} \boldsymbol{\eta}_{i}(t)\right),
$$

where $G_{0}$ is the instantaneous shear modulus, $g_{i}$ are dimensionless relaxation shear coefficients and

$$
\eta_{i}(t)=\int_{0}^{t}\left(1-e^{(\tau-t) / \tau_{i}}\right) \dot{\eta}(\tau) d \tau=\frac{1}{\tau_{i}} \int_{0}^{t} e^{-s / \tau_{i}} \eta(t-s) d s .
$$

The summation in eq. (6) is the deviatoric "creep" strain, $\eta^{c r}(t)$, that is, the difference between the total strain and the instantaneous elastic strain, $S(t) / 2 G_{0}$.

Similar remarks apply to the viscoelastic volumetric behaviour of the material.

The creep model described above is implemented in the commercial finite element code Abaqus, which was used in the applications presented in Sections 3 and 4. Details about the numerical integration of eq. (6) can be found in [4]. 


\subsection{Experimental results}

To illustrate the creep behaviour of masonry and its constituents, reference will be made to the results carried out by Brooks [5] and reported in [6] together with a theoretical prediction of the creep curves obtained from tests on masonry walls.

Calcium silicate bricks were subjected to a sustained stress of $3 \mathrm{MPa}$ for 300 days, whereas Portland cement and lime sand mortar specimens were tested for a similar period under a stress of $3.4 \mathrm{MPa}$. The results obtained are reported in Fig. 1, together with a best fit of the experimental points by Prony series, eq. (3), of one or two terms. The values of the parameters defining the theoretical creep curves are listed in Table 1: the volumetric creep behaviour of bricks and mortar is assumed to be defined by the same non-dimensional coefficients $g_{i}$ that define their deviatoric creep behaviour (see eq. (6)).

(a)

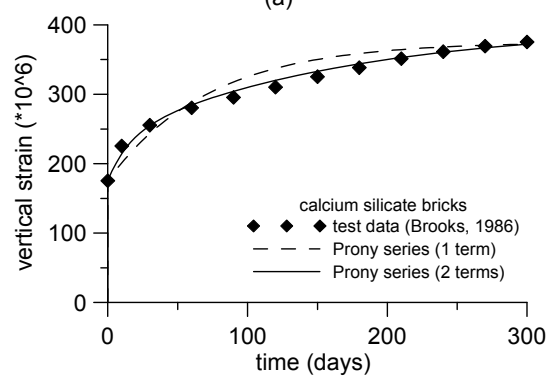

(b)

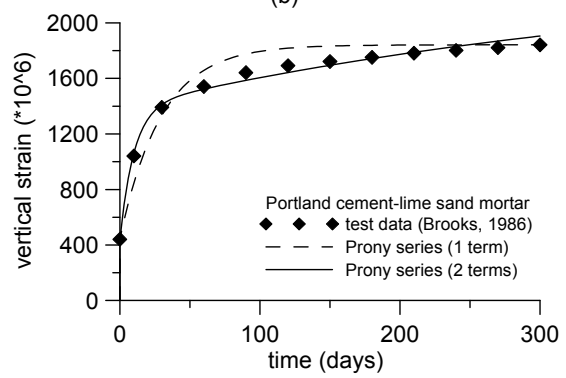

Figure 1: Creep tests on (a) calcium silicate bricks and (b) Portland cementlime sand mortar: experimental tests [5] vs. theoretical modelling by Prony series.

Table 1: Parameters defining the creep behaviour of bricks and mortar (eq. (6)) employed in the numerical simulations.

\begin{tabular}{|c|c|c|c|}
\cline { 2 - 4 } \multicolumn{2}{c|}{} & brick & mortar \\
\hline \multicolumn{2}{|c|}{$E_{0}[\mathrm{MPa}]$} & 17100 & 7700 \\
\hline \multicolumn{2}{|c|}{$G_{0}[\mathrm{MPa}]$} & 7435 & 3208 \\
\hline \multirow{3}{*}{$n=1$} & $g_{1}$ & 0.5327 & 0.7602 \\
\cline { 2 - 4 } & $\tau_{1}[$ days] & 33.8 & 7.1 \\
\hline \multirow{4}{*}{$n=2$} & $g_{1}$ & 0.285 & 0.690 \\
\cline { 2 - 4 } & $\tau_{1}$ [days] & 11.1 & 3.1 \\
\cline { 2 - 4 } & $g_{2}$ & 0.275 & 0.125 \\
\cline { 2 - 4 } & $\tau_{2}$ [days] & 100.1 & 243.1 \\
\hline
\end{tabular}


It can be noted that using Prony series of two terms, rather than one term, definitely improves the match with the experimental points. In particular, a best fit with two terms captures a primary creep phase of a duration longer than the creep test itself, conforming with the experimental data.

A single-leaf wall made of the same constituents was also subjected to a creep test of a duration of 300 days under a vertical stress of $3 \mathrm{MPa}$ : the experimental results reported in [6] are shown in Fig. 2. These results will be used in Sec. 3.2 to validate the proposed finite element models.

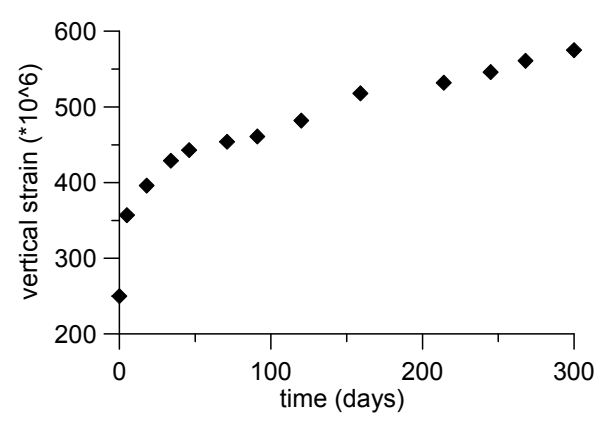

Figure 2: $\quad$ Creep tests on a single-leaf wall made of calcium silicate bricks and Portland cement-lime sand mortar [6].

\section{Numerical simulation of creep tests on masonry walls}

\subsection{Finite element models of brick masonry walls}

The creep behaviour of brickwork was numerically investigated using three different finite element models: a simplified two-dimensional (layered) model (Fig. 3(a)) and two more detailed three-dimensional models reproducing a header bond brickwork (Fig. 3(b)) and a Flemish bond brickwork (Fig. 3(c)). The layered model consists of thirteen $65 \mathrm{~mm}$-thick brick courses, alternated with 12 mm-thick mortar bed joints, thus matching the geometry of the brickwork subjected to the creep test shown in Fig. 2 [6]. The 3D models consist of 20 brick courses: the size of the units is $55 \times 120 \times 250 \mathrm{~mm}$; bed and head mortar joints have a thickness of $10 \mathrm{~mm}$. The mortar-to-brick thickness ratio is 0.18 both in the $2 \mathrm{D}$ and in the 3D models. 4-node and 8-node isoparametric elements, with a bilinear discretization of the displacement field, were employed in the $2 \mathrm{D}$ and in the $3 \mathrm{D}$ analyses, respectively.

In the applications, the self-weight of the materials is disregarded, as it was numerically found to have negligible effects on the results. The models are subjected to a uniform vertical stress of $3 \mathrm{MPa}$ at the top side; the lower base is fully restrained.

Simulations of creep tests of a duration of 300 days using creep laws described by Prony series of one or two terms were carried out. The material parameters employed in the numerical applications are those listed in Table 1. 


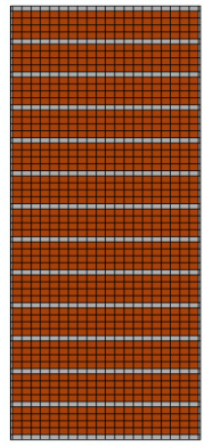

(a)

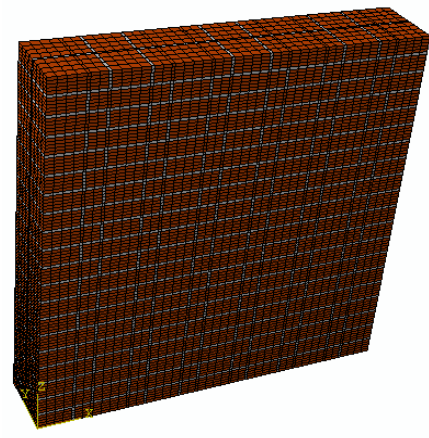

(b)

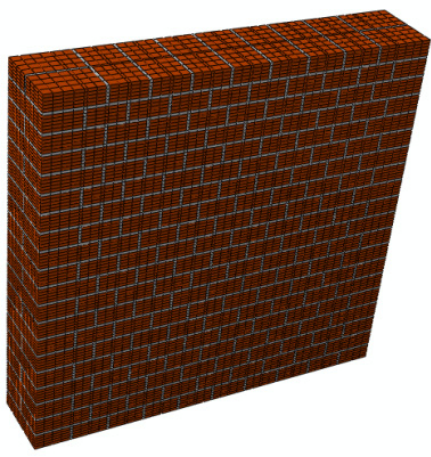

(c)

Figure 3: Finite elements employed in the numerical applications: (a) simplified 2D layered model; (b) 3D Flemish bond brickwork model; (c) 3D header bond brickwork model.

\subsection{Creep curves}

The results of the simulation of the creep test shown in Fig. 2 using the 2D layered model of Fig. 3(a) are shown in Fig. 4, where the vertical displacement of the central node of the top side is plotted vs. time. Again, using Prony series of two terms to describe the creep behaviour of bricks and mortar definitely improves the fit with the experimental data also when the global response of brickwork has to be predicted.

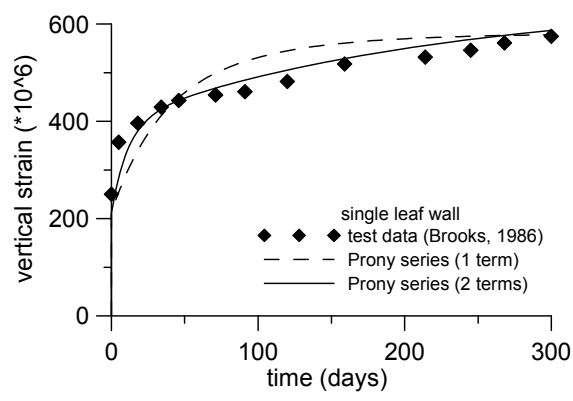

Figure 4: Creep tests on a singleleaf wall: experimental results [6] vs. predictions of the 2D FE model.

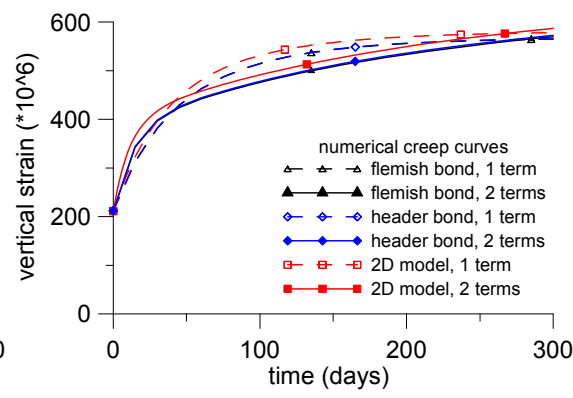

Figure 5: Simulation of creep tests on a single-leaf wall with different numerical models.

The same creep test is numerically simulated using the 3D models shown in Figs. 3(b), (c), both using one term and two terms in the Prony series for the creep laws of the constituents. The results obtained are shown in Fig. 5. Apparently, the difference between the Flemish bond model and the header bond model is negligible when the same number of terms in the creep law is used. The 
simplified 2D model and the 3D models predict the same time evolution, the 2D model being slightly more deformable than the $3 \mathrm{D}$ ones.

\section{Stress evolution}

It is instructive to follow the time evolution of the stress components in typical regions of a masonry wall, according to the different numerical models employed in the present work (see Fig. 3). Only creep laws consisting of Prony series of two terms are considered.

Fig. 6 shows the distribution of the vertical stress in the 3D models at the beginning of the application of the load. The stress fluctuation induced by the brickwork heterogeneity is apparent: this fluctuation cannot be captured by the simplified layered model, which obviously predicts a uniform vertical stress of $3 \mathrm{MPa}$ in both layers throughout the entire load history. The vertical stress tends to flow across the bricks bypassing the softer head joints.

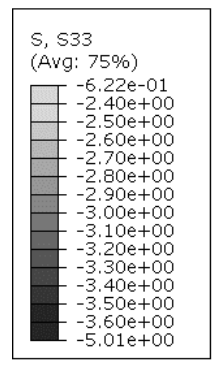

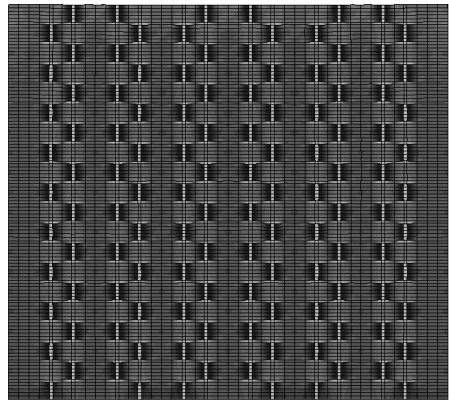

(a)

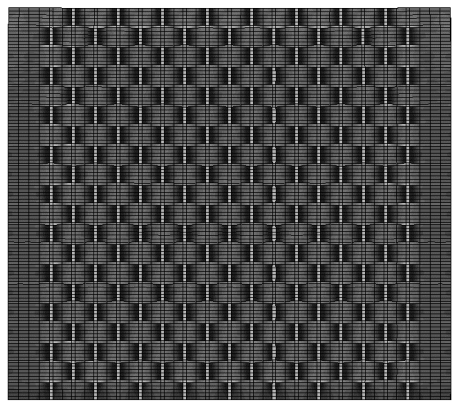

(b)

Figure 6: Vertical stress in (a) Flemish bond and (b) header bond brickwork under a uniform vertical load of $3 \mathrm{MPa}$ at $t=0$.

Fig. 7 shows the pattern of the transversal stress (i.e., the normal stress component parallel to the mortar beds) over the 3D models at the application of the vertical load. As is well known, owing to Poisson's effect bed joints are compressed and bricks are mostly in tension parallel to the mortar beds. The highest tensile stresses are encountered close to the head joints, which are mainly subjected to transverse tension too: these stresses might be responsible for the failure of the brick/mortar interface along these joints.

Plots of specific stress and strain components versus time, computed at different points of the models, are shown in Figs. 9 to 11. All the plots refer to points in the brickwork sufficiently far from the model boundaries. As far as the layered model is concerned, points located on the mid-section of the model in the brick and in the mortar layers will be considered. The points where stresses and strains are computed in the 3D models are shown in Fig. 8. These include points located in stretchers $\left(s_{1}, s_{2}\right)$, headers $\left(h, h_{1}, h_{2}\right)$, bed joints $\left(b j, b j_{1}, b j_{2}\right)$ and head joints (hj). Points near the outer face of the wall (ext), as well as near the wall mid-surface (int), are considered. 


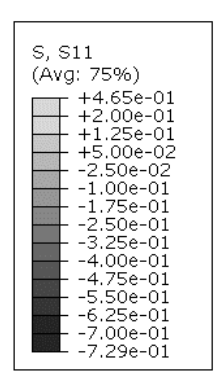

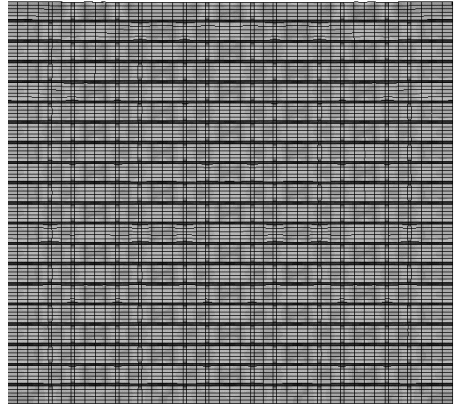

(a)

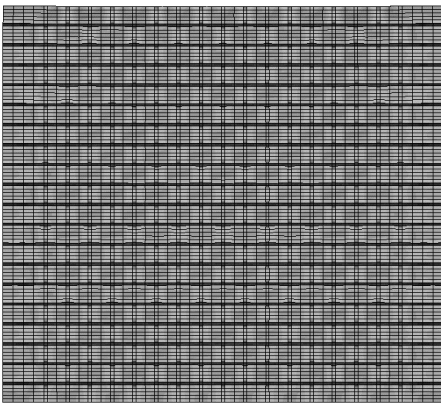

(b)

Figure 7: Transversal stress in (a) Flemish bond and (b) header bond brickwork under a uniform vertical load of $3 \mathrm{MPa}$ at $t=0$.

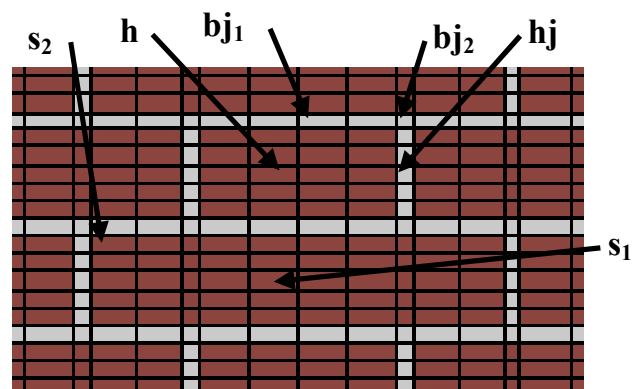

(a)

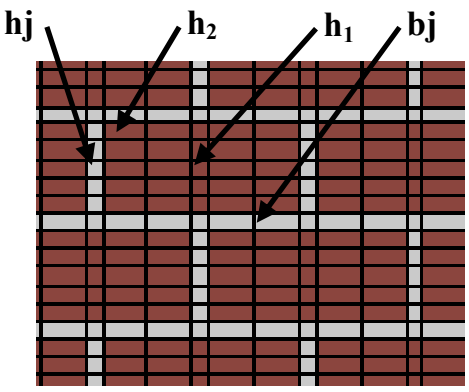

(b)

Figure 8: $\quad$ Points where stresses and strains are computed in (a) Flemish bond brickwork and (b) header bond brickwork.

\subsection{Flemish bond brickwork}

The time evolution of the vertical (axial) stress in selected points of Flemish bond brickwork is shown in Figs. 9(a) and (b): Fig. 9(a) refers to the points located in the bricks, whereas Fig. 9(b) to points located in the bed and head joints. Note that the time evolution of the stress in most points is not monotonic: the stress attains a maximum (or a minimum) nearly 30 days after loading, and takes values close to the long-term ones at nearly 100 days after loading.

The 2D layered model underestimates the axial stress in the bricks (Fig. 9(a)), except for the innermost region of the headers (curve $h_{1}$,int). The highest axial stresses are attained at the shortest sides of the stretchers, especially at the interior of the wall (curve $s_{2}$,int). The difference between the short-term predictions of the $2 \mathrm{D}$ model and the $3 \mathrm{D}$ model ranges between $2-3 \%$ in the headers (points $h_{1}$,int and $h_{1}$, ext) and $9-11 \%$ at the shortest sides of the stretchers (points $s_{2}$,int and $s_{2}$,ext). These differences increase in time: the highest 
discrepancy 300 days after loading is attained at the vertices of the stretchers (point $\mathrm{s}_{2}$,int), and is of the order of $20 \%$ of the stress predicted by the $2 \mathrm{D}$ model.

On the contrary, the layered model overestimates the axial stress in most regions of the mortar joints (Fig. 9(b)), except for the bed joints (curve bj,ext). In particular, the axial stress predicted by the $2 \mathrm{D}$ model is nearly twice that computed in the head joints in the short term (points hj,int and hj,ext), and the long-term prediction is only $27 \%$ of the applied stress.

The main differences between the predictions given by the $2 \mathrm{D}$ model and the 3D model of Flemish bond brickwork are observed in terms of transversal stresses, which are plotted in Fig. 10 versus time. Fig. 10(a) refers to points located in bricks; Fig. 10(b), to points located in mortar. Contrary to the axial stress, the transversal stress predicted by the layered model varies in time, and ranges between 0.02 and $0.06 \mathrm{MPa}$ in the brick layer and between -0.1 and $-0.32 \mathrm{MPa}$ in the mortar layer during the numerical creep test.

The 2D model underestimates the tensile transversal stress in most regions of the stretchers and overestimates the stress in the headers (Fig. 10(a)). The highest computed stresses in the stretchers (curve $s_{2}$,int) vary between $0.13 \mathrm{MPa}$ at $t=0$

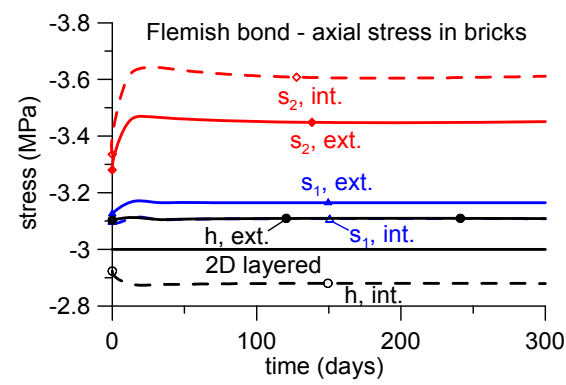

(a)

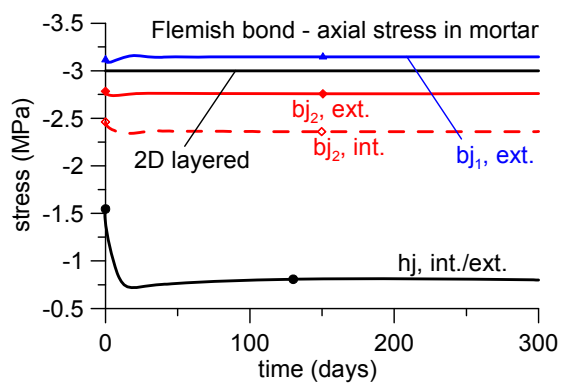

(b)

Figure 9: Time evolution of the axial stress in Flemish bond brickwork: (a) bricks; (b) mortar.

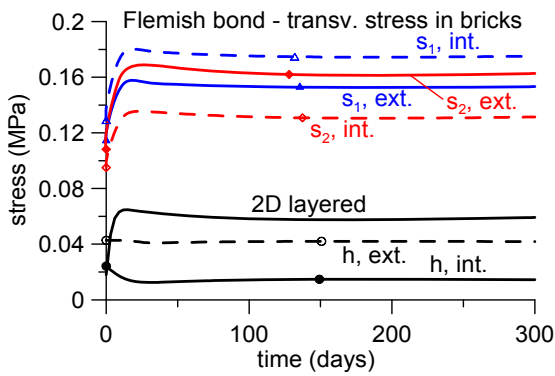

(a)

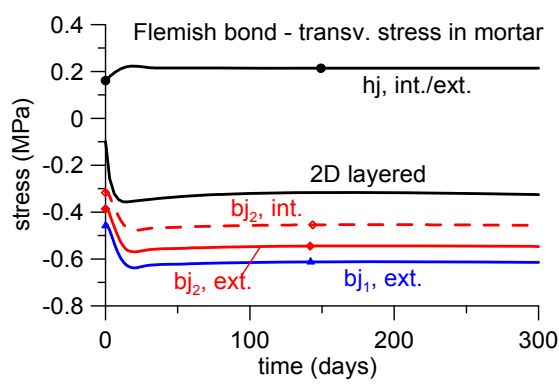

(b)

Figure 10: Time evolution of the transversal stress in Flemish bond brickwork:

(a) bricks; (b) mortar. 
and $0.18 \mathrm{MPa}$ at $t=300$ days, attaining non-negligible values contrary to the predictions of the $2 \mathrm{D}$ model.

The compressive transversal stress in the mortar joints is underestimated by the 2D model: the highest compressions are found in the bed joints (curve $b_{1}$, int in Fig. 10(b)) and vary in time from -0.46 to $-0.61 \mathrm{MPa}$. As already remarked, the transversal stress computed in the head joints is tensile (curve hj,int/ext in Fig. 10(b)), and might cause debonding at the interface between bricks and head joints.

\subsection{Header bond brickwork}

Fig. 11(a) shows the time evolution of the axial stress in the points of the header bond brickwork indicated in Fig. 8(b). The time evolution of the stress in most points is not monotonic, similarly to Flemish bond brickwork. The 2D layered model predicts axial stresses very close to those computed in the bed joints of the 3D model (curve bj in Fig. 11(a)), and intermediate between the stresses computed at the center (curve $h_{1}$ ) and at the vertices (curve $h_{2}$ ) of the headers. At the center, the axial stress is lower than the applied stress by a 3\% in the short term, and by $5 \%$ in the long term. At the vertices, the axial stress exceeds by $9 \%$ the applied stress at the beginning of the creep test; it furtherly increases in the long term, exceeding by $15 \%$ the applied stress. The layered model definitely overestimates the stress in the head joints (curve hj), that varies between half of the applied stress at $t=0$ and $27 \%$ of the applied stress in the long term.

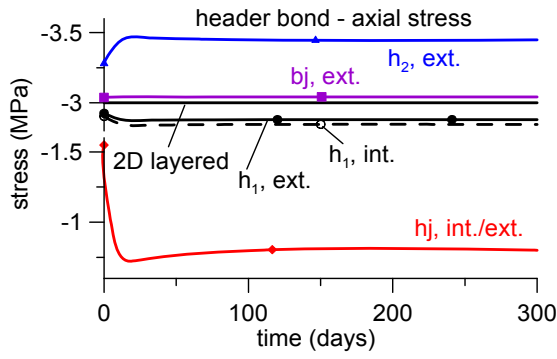

(a)

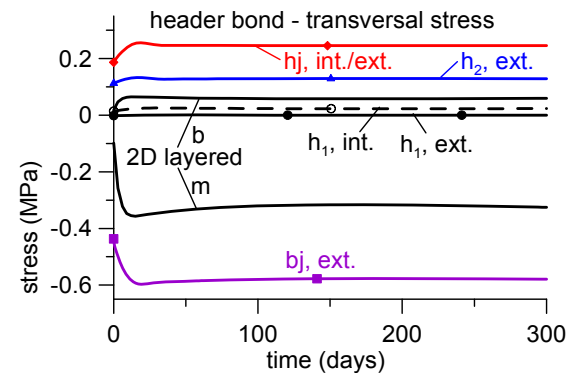

(b)

Figure 11: Stress evolution in header bond brickwork: (a) vertical stress and (b) transversal stress.

The time evolution of the transversal stress in selected points of the header bond brickwork model is shown in Fig. 11(b). The stress predicted by the 2D model in the bricks falls between those computed at the center (curves $h_{1}$,int, $\mathrm{h}_{1}$, ext) and at the vertices (curve $\mathrm{h}_{2}$ ) of the headers. Similarly to the case of Flemish bond brickwork, the 2D model definitely underestimates the compressive stress in the bed joints (curve bj): the stress predicted by the layered model is nearly half that computed by the 3D model in the long term. Again, the transversal stress in the head joints is tensile (curve hj,int/ext in Fig. 11(b)), of the same order of magnitude of that computed in Flemish bond brickwork. 
Finally, note that, owing to its simpler pattern, in header bond brickwork stresses are more uniform across the wall thickness than in Flemish bond brickwork (compare curves $h_{1}$, int and $h_{1}$,ext in Figs. 11(a) and 11(b)).

\section{Concluding remarks}

The purpose of the study presented in this paper was twofold: on the one hand, assessing whether the creep behaviour of brickwork could be predicted using a simplified layered two-dimensional model rather than sophisticated 3D models; on the other hand, analysing the influence of the brick pattern on the distribution of the stresses and their redistribution in time under a constant load.

According to the comparisons discussed Sec. 3, it can be stated that (a) the layered model predicts the experimental global creep strain of a single-leaf wall with fair accuracy, provided that two terms are employed in the Prony series that describe the creep behaviour of the individual components (see Fig. 4); (b) the layered model and the three-dimensional models of two different brickworks basically predict the same axial creep strains (see Fig. 5). This indicates that neither the brick pattern, nor the head joints, affect the global deformation of the wall significantly.

On the contrary, the differences in terms of vertical stresses and, even more, horizontal stresses predicted by the $2 \mathrm{D}$ model and the $3 \mathrm{D}$ models are very pronounced. This is basically due to the fluctuation of stresses (and strains) from one component to another in the same layer because of the presence of the head joints that cannot be captured by the layered model. Using the simplified 2D model might give acceptable predictions of the axial stress in the headers of the analyzed brickworks and in the bed joints of header bond brickwork, but the axial stress in the stretchers and in the bed joints of Flemish bond brickwork is heavily underestimated. The transversal stresses predicted by the $2 \mathrm{D}$ model are mostly unreliable. Debonding is likely to occur at the interface between bricks and head joints according to the high tensile stresses computed. To predict the time evolution of the stresses across any brickwork with more accuracy, the inability of the head joints to transfer transversal stresses should be taken into account.

\section{Acknowledgements}

The author is grateful to MM Gabriele Banfi and Pietro Gagliardi for their assistance in the finite element modelling and data processing.

\section{References}

[1] Taliercio, A., An overview of masonry creep, in 'Structural Studies, Repairs and Maintenance of Heritage Architecture XI', C.A. Brebbia (Ed.), WIT Press, Southampton (GB), 2009, pp. 197-208. 
682 Structural Repairs and Maintenance of Heritage Architecture XII

[2] Park, S.W., Schapery, S.A., Methods of interconversion between linear viscoelastic material functions. Part I-a numerical method based on Prony series, Int. J. Solids Struct., 36(11), pp. 1653-1675, 1999.

[3] Kaliske, M., Rothert, H., Formulation and implementation of threedimensional viscoelasticity at small and finite strains, Comput. Mech., 19(3), pp. 228-239, 1997.

[4] ABAQUS Theory Manual, Version 6.8-1.

[5] Brooks, J.J., Composite models for predicting elastic and long-term movements in brickwork walls, Proc. Br. Masonry Soc., 1, pp. 20-23, 1986.

[6] Brooks, J.J., Composite modelling of masonry deformation, Materials and Structures RILEM, 23, pp. 241-251, 1990. 